\title{
School Rehabilitation Program's (SRP) Contribution to School Management and Students' Academic Performance to Beneficiary Schools in Cebu Province, Philippines
}

\author{
Aurora Q. Pestaño ${ }^{1}$, Dwight J. Ypanto ${ }^{2}$, Ricky B. Villeta ${ }^{3}$, Milagros B. Baclayon ${ }^{4}$, Chanine F. Sevilla ${ }^{4}$, Jessica M. \\ Avenido $^{4}$, Jesse S. Susada ${ }^{4}$ \\ ${ }^{1}$ College of Arts and Sciences-Department of Psychology and Library Information Sciences, University of San Jose \\ Recoletos, Cebu City, Philippines \\ ${ }^{2}$ Center for Religious Education, University of San Jose Recoletos, Cebu City, Philippines \\ ${ }^{3}$ College of Arts and Sciences-Department of Mathematics and Sciences, University of San Jose Recoletos, Cebu City, \\ Philippines \\ ${ }^{4}$ Center for Publication Development and Research Studies, University of San Jose Recoletos, Cebu City, Philippines \\ Correspondence: Aurora Q. Pestaño, College of Arts and Sciences-Department of Psychology and Library Information \\ Sciences, University of San Jose Recoletos, Cebu City, Philippines.
}

Received: September 9, 2018

doi:10.11114/ijce.v1i2.3608
Accepted: October 11, $2018 \quad$ Online Published: October 16, 2018

URL: https://doi.org/10.11114/ijce.v1i2.3608

\begin{abstract}
In an educational setting, the management and environment are bound to affect the whole learning environment. This study determines the impact of the Ramon Aboitiz Foundation Incorporated School Rehabilitation Program (RAFI-SRP) to beneficiary schools. The Descriptive-correlational design is utilized to establish the level of assessments for school buildings regarding its milieu, environment, management, and students' academic performance during their occupancy of the RAFI classrooms. In the exploratory data analyses, multivariate principal component analysis (PCA) and regression are used. Results show that RAFI-SRP affects school management. The more new school buildings are built, the better is the performance of the school administrators. Moreover, RAFI-SRP has a modest influence on the academic performance of students among the community beneficiaries as a whole. This result may be owed to the fact that academic performance is multifactorial, such that the interplay of factors may be intrinsic and extrinsic. RAFI-SRP is just one amongst the extrinsic factors to the learners' academic performance to contend with.
\end{abstract}

Keywords: RAFI-SRP, school management, school milieu, school environment, students' academic performance, descriptive, multivariate principal component, regression analysis

\section{Introduction}

An organization's efficacy depends on its environment as well as the people who compose it. The management and environment perform a part in orchestrating the roles, performances, and achievements of those who are led and managed. In an educational setting, the management and environment are bound to affect the whole organization whose primary responsibility is to implement the school's goals and objectives through the exercise of sound school management and functional learning environment that includes the school milieu and school environment. If these prerequisites are present, the school management and the learning environment can serve the purposes for which they operate and deliver the services which they aim to offer.

In the study of Yeoman (2012), he claims that it is difficult to raise the level of academic achievement when surroundings are decrepit. Hence, the physical school environment is an area that administrators need to focus on: location, size of a classroom, furniture, lighting, temperature, ventilation, noise level, and the like. The theory of Theo Dalton (1951) posited that the classroom atmosphere reflects quality learning. By classroom atmosphere, he speaks of lighting, ventilation, heating, cleanliness, and orderliness of the classroom, behavior, and attitude of the teacher towards the student and the attitude and character of students towards one another.

Many authors have had studies on the influence of school buildings on the learning performances of students in the past. 
However, the connection and links have not been studied at length.

In this particular study, the level of assessments for school buildings, milieu, school environment, and school management was correlated, regressed with the academic performance of students to find out if indeed school buildings influenced the students' learning performances.

Students, teachers' performance and satisfaction, are influenced by the condition of the school milieu (Cash \& Twiford, 2010). The general classroom atmosphere and environment impacts learning as such that a clean and orderly classroom facilitates focus, concentration, and comfort. It also promotes mental hygiene. It was also shown that the physical condition of the building affects teachers' morale, sense of safety and the feeling of effectiveness in the classroom (Corcoran, T.B., Walker, L.J., and White, J.L., 1988). The theory of Theo Dalton postulates that well-lighted classroom is very conducive for reading, writing, and other activities. Seemingly small details like lighting impact student performance. Estimates vary from .3 percent to 26 percent as to how much increase in student performance is directly attributable to lighting condition (Baker and Bernstein, 2012). A classroom that is well ventilated also renders the same effect.

Lave and Wenger (1990) argue that learning is situated; that is, as it normally occurs, learning is embedded within activity and context. It is also usually unintentional rather than deliberate. Knowledge needs to be presented in authentic contexts - settings and situations that would normally involve that knowledge. Social interaction and collaboration are essential components of learning where learners become involved which embodies certain beliefs and behaviors to be acquired. In likewise manner, failure to invest in school milieu sends a wrong signal to students and affects their morale.

Studies on the relationship between satisfaction with the learning environment and students' efforts, on the other hand, pointed out that physical work conditions is of less importance but can stimulate students' effort (Hopland \& Nyhus, 2016). The cancellation of the Building-Schools-for-the-Future Program in the US in 2010 and the establishment of the James Review is worth noting. The review pointed to a waste of money in the building projects and the needed assessment of the state of school milieus in the country (Piggott, 2012).

The Ramon Aboitiz Foundation Incorporated (RAFI) is one institution that invests in public school rehabilitation program. It has annually donated materials, manpower, project management among others in the reconstruction and rehabilitation of public school classrooms and buildings throughout the province of Cebu, since the advent of typhoon Haiyan in 2013. This program is dubbed as School Rehabilitation Program (SRP). Since then and until 2016, RAFI was able to rehabilitate/reconstruct around 1,650 classrooms in 450 schools. However, no formal study has been made to evaluate the impact, relevance, and sustainability of the said project.

\section{Framework of the Study}

This study sought to determine the impact of the School Rehabilitation Program (SRP) of RAFI to its community beneficiaries and the locals in the community as a whole. In this study, school management refers to leadership and governance, curriculum and learning, accountability and continuous improvement as well as the management of resources. School milieu, on the other hand, refers to context, massing, interface, wayfinding, social space and comfort building elements. While school environment includes physical features, outdoor areas, learning environments, social areas, media access, transition spaces and circulation routes, visual appearance, and safety and security. More often, milieu and environment are used interchangeably, but in this particular study, the terms are technically defined as fore mentioned. The academic performance of the students using these classrooms was also one of the factors considered.

The impact of factors affecting school milieu, school environment and school management are modeled to determine and characterize their contribution. The impact of school milieu and school environment on school management is further established to determine the level of the effect they have on school administration as perceived by school administrators and teachers. Consequently, the possible impact of school milieu, school environment and school management on learners' academic performance are determined to establish the impact of the RAFI-SRP. 


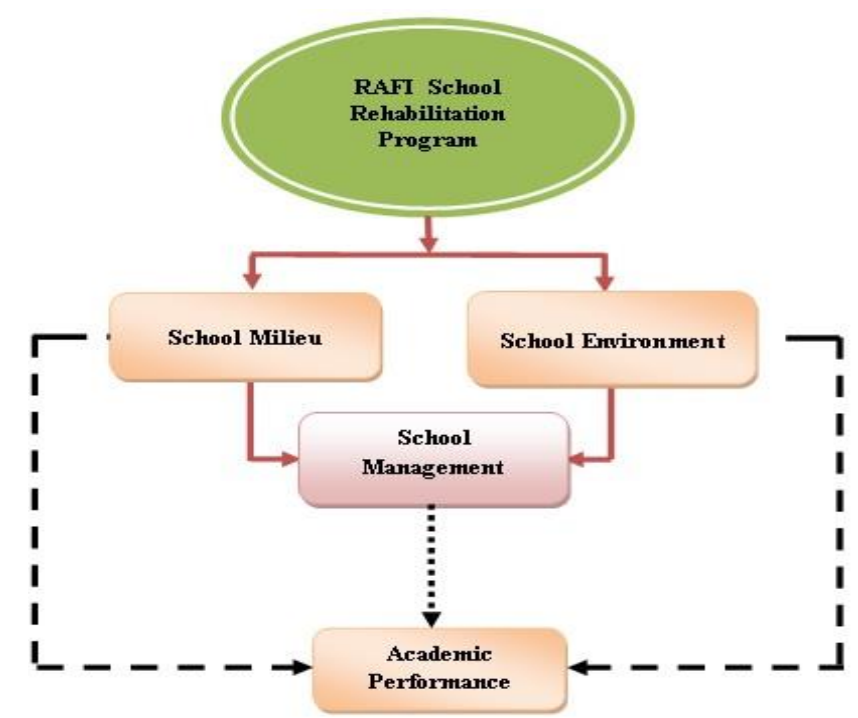

Figure 1. Framework of the Study

\section{Design and Methods}

This study utilized the descriptive-correlational survey method. Qualitative data, on the other hand, were utilized to substantiate the results and findings of the study. The validated researcher-made questionnaires were administered to ascertain the level of impact of the project on the beneficiary and the community. The reliability of the questionnaires and instruments are found highly sufficient regarding statistical internal consistency among indicators of the dimensions involved in the instrumentation development process. Descriptive and exploratory methods of analyses using multivariate principal component and multiple regression are used in the study. For qualitative data, an interview schedule was utilized.

Cochran's (1977) formula for the continuous variable (Bartlett, J.E., Kotrlik, J.W. \& Higgins, C.C. (2001) was applied to determine the required number of elementary and secondary schools in the province of Cebu, with classroom rehabilitated/reconstructed/constructed by RAFI to be included as research respondents and locale for this study. The sample size determination considers the five (5) points scale adopted in the questionnaire instrument, with the level of significance set at 0.05 that gives at-value of 1.96 for the selected alpha of 0.025 in each tail, an acceptable margin of error of $3 \%$, and an estimated standard deviation of 1.25 . The formula is given below.

$$
n=\frac{t^{2} * s^{2}}{d^{2}}
$$

where:

$$
\begin{aligned}
& t=\text { value for the selected alpha of } 0.025 \text { in each tail of } 1.96 \\
& s=\text { estimate of standard deviation in the population of } 1.25 \\
& d=\text { acceptable margin of error for the mean is estimated as } 0.15 .
\end{aligned}
$$

Given the population size of 450 schools that have benefitted the reconstruction and rehabilitation program by RAFI since 2004 to 2016, a total of 148 public schools from the different congressional districts in the province of Cebu comprised the required number of samples needed in the study. The sampling proportion was further applied to determine the appropriate number of schools per district necessary to participate in the study. The following determines the sampling proportion formula.

$$
\text { Desired sample size }
$$

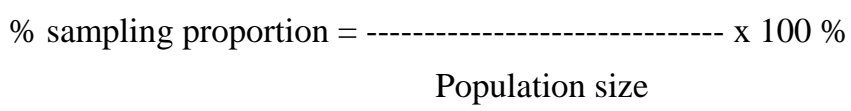

The resulting common sampling proportion was employed to calculate the number of schools to be taken from the respective districts. Subsequently, multistage sampling was employed to generate random respondents comprising the school administrators, teachers, students and parents in selecting samples for the study. The selection of classrooms rehabilitated/reconstructed/constructed by RAFI in randomly selected schools as well as the students and parents in the 
study made use of systematic and random sampling processes done using the sampling software.

The study utilized three research instruments. These instruments underwent face validation, dry run, and reliability testing before its usage to actual respondents.

To assess the school management of key persons like principals, head teachers, and teachers in the selected schools, researchers adapted and modified the Revised School-Based Management (SBM) Assessment tool. The tool was utilized to classify school management systems as beginning, developing or advanced (accredited level) (Department of Education, 2012).

Henry Sanof's Six Factor School Building Checklist (2011) was adapted and modified to measure school milieu. The six-factor school building assessment instrument for the school milieu focused on six key elements of building assessment --- context (school building setting), massing(parts of the building that gives it form, meaning and variety), interface(meeting place where the inside of the building connects with the outside), wayfinding(ability for students, staff, teachers, and visitors to discern routes, traffic patterns or passageways in or around the building), social space and comfort(ability of the school environment to accommodate diverse human needs). A series of checklist questions and a numerical rating scale were designated for each factor being assessed.

The third instrument is the modified School Building Rating Scale used to measure school environment (Sanoff, 2011). The components of the rating scale for the school environment include physical features, outdoor areas, learning environments, social areas, media access, transition spaces and circulation routes, visual appearance, and safety and security.

To determine the impact of RAFI's SRP to the beneficiaries, student's academic performance was utilized as one dependent variable.

\section{Results and Discussion}

This section presents the findings of the study from the dimensions of school management that have been improved with RAFI's SRP directly or indirectly.

Presentation on the findings regarding the extent of learning environment that includes school milieu and school environment follows thereafter. The findings for the academic performance of students are discussed in this section.

Overall Assessment of Factors Affecting the School Management, School Milieu, and School Environment as Perceived by the Respondents among Schools across Six Districts in the Province of Cebu 
Table 1.Overall Extent on School Management among Schools across Six Congressional Districts

\begin{tabular}{|c|c|c|c|c|c|c|c|c|}
\hline \multirow{2}{*}{$\begin{array}{c}\text { School } \\
\text { Management } \\
\text { Factors }\end{array}$} & \multirow[t]{2}{*}{$\begin{array}{l}\text { Congressional } \\
\text { Districts }\end{array}$} & \multicolumn{2}{|c|}{$\underset{(n=68)}{\text { Administrators }}$} & \multicolumn{2}{|c|}{$\begin{array}{l}\text { Teachers } \\
(n=389)\end{array}$} & \multirow[t]{2}{*}{ Reliability } & \multirow[t]{2}{*}{$\begin{array}{l}\text { Overall } \\
\text { Mean }\end{array}$} & \multirow[t]{2}{*}{ Interpretation } \\
\hline & & Mean & Stdev & Mean & Stdev & & & \\
\hline \multirow[t]{6}{*}{$\begin{array}{l}\text { Leadership and } \\
\text { Governance }\end{array}$} & District 1 & 4.08 & 0.15 & 4.20 & 0.06 & 0.9228 & 4.14 & Practices and procedure satisfy quality \\
\hline & District 2 & 4.00 & 0.15 & 4.23 & 0.05 & 0.9140 & 4.12 & $\begin{array}{c}\text { Practices and procedure satisfy quality } \\
\text { standard }\end{array}$ \\
\hline & District 3 & 3.62 & 0.15 & 4.34 & 0.07 & 0.9617 & 3.98 & $\begin{array}{c}\text { Practices and procedure satisfy quality } \\
\text { standard }\end{array}$ \\
\hline & District 4 & 4.20 & 0.10 & 4.11 & 0.04 & 0.9545 & 4.16 & $\begin{array}{c}\text { Practices and procedure satisfy quality } \\
\text { standard }\end{array}$ \\
\hline & District 5 & 4.01 & 0.09 & 4.22 & 0.06 & 0.9536 & 4.11 & $\begin{array}{c}\text { Practices and procedure satisfy quality } \\
\text { standard }\end{array}$ \\
\hline & District 7 & 3.82 & 0.34 & 3.75 & 0.13 & 0.9228 & 3.79 & $\begin{array}{c}\text { Practices and procedure satisfy quality } \\
\text { standard }\end{array}$ \\
\hline \multirow[t]{6}{*}{$\begin{array}{l}\text { Curriculum } \\
\text { Learning }\end{array}$} & District 1 & 4.10 & 0.21 & 4.16 & 0.06 & 0.9033 & 4.13 & $\begin{array}{c}\text { Practices and procedure satisfy quality } \\
\text { standard }\end{array}$ \\
\hline & District 2 & 3.98 & 0.28 & 4.22 & 0.07 & 0.9128 & 4.10 & $\begin{array}{c}\text { Practices and procedure satisfy quality } \\
\text { standard }\end{array}$ \\
\hline & District 3 & 3.77 & 0.20 & 4.34 & 0.11 & 0.9073 & 4.06 & $\begin{array}{c}\text { Practices and procedure satisfy quality } \\
\text { standard }\end{array}$ \\
\hline & District 4 & 4.04 & 0.18 & 4.02 & 0.13 & 0.8961 & 4.03 & $\begin{array}{c}\text { Practices and procedure satisfy quality } \\
\text { standard }\end{array}$ \\
\hline & District 5 & 3.91 & 0.30 & 4.16 & 0.09 & 0.9368 & 4.03 & $\begin{array}{c}\text { Practices and procedure satisfy quality } \\
\text { standard }\end{array}$ \\
\hline & District 7 & 4.20 & 0.15 & 3.81 & 0.23 & 0.8929 & 4.01 & $\begin{array}{c}\text { Practices and procedure satisfy quality } \\
\text { standard }\end{array}$ \\
\hline \multirow{6}{*}{$\begin{array}{l}\text { Accountability and } \\
\text { Continuous } \\
\text { Improvement }\end{array}$} & District 1 & 3.80 & 0.22 & 4.08 & 0.05 & 0.9216 & 3.94 & $\begin{array}{c}\text { Practices and procedure satisfy quality } \\
\text { standard }\end{array}$ \\
\hline & District 2 & 3.75 & 0.50 & 4.11 & 0.06 & 0.8845 & 3.93 & $\begin{array}{c}\text { Practices and procedure satisfy quality } \\
\text { standard }\end{array}$ \\
\hline & District 3 & 3.61 & 0.05 & 4.26 & 0.10 & 0.9036 & 3.94 & $\begin{array}{c}\text { Practices and procedure satisfy quality } \\
\text { standard }\end{array}$ \\
\hline & District 4 & 3.96 & 0.28 & 3.93 & 0.05 & 0.8625 & 3.95 & $\begin{array}{c}\text { Practices and procedure satisfy quality } \\
\text { standard }\end{array}$ \\
\hline & District 5 & 3.65 & 0.10 & 4.11 & 0.08 & 0.9323 & 3.88 & $\begin{array}{c}\text { Practices and procedure satisfy quality } \\
\text { standard }\end{array}$ \\
\hline & District 7 & 3.92 & 0.34 & 3.98 & 0.14 & 0.8932 & 3.95 & $\begin{array}{c}\text { Practices and procedure satisfy quality } \\
\text { standard }\end{array}$ \\
\hline \multirow[t]{6}{*}{$\begin{array}{l}\text { Management of } \\
\text { Resources }\end{array}$} & District 1 & 3.90 & 0.15 & 4.16 & 0.04 & 0.8811 & 4.03 & $\begin{array}{c}\text { Practices and procedure satisfy quality } \\
\text { standard }\end{array}$ \\
\hline & District 2 & 3.88 & 0.33 & 4.17 & 0.08 & 0.8920 & 4.02 & $\begin{array}{c}\text { Practices and procedure satisfy quality } \\
\text { standard }\end{array}$ \\
\hline & District 3 & 3.60 & 0.07 & 4.32 & 0.15 & 0.8877 & 3.96 & $\begin{array}{c}\text { Practices and procedure satisfy quality } \\
\text { standard }\end{array}$ \\
\hline & District 4 & 4.00 & 0.10 & 3.98 & 0.06 & 0.9198 & 3.99 & $\begin{array}{c}\text { Practices and procedure satisfy quality } \\
\text { standard }\end{array}$ \\
\hline & District 5 & 3.78 & 0.26 & 4.15 & 0.03 & 0.9053 & 3.97 & $\begin{array}{c}\text { Practices and procedure satisfy quality } \\
\text { standard }\end{array}$ \\
\hline & District 7 & 4.16 & 0.17 & 3.91 & 0.11 & 0.9356 & 4.04 & $\begin{array}{c}\text { Practices and procedure satisfy quality } \\
\text { standard }\end{array}$ \\
\hline
\end{tabular}

Legend:

\section{Mean Range}

$\begin{array}{lll}4.20 & - & 5.00 \\ 3.40 & - & 4.19 \\ 2.60 & - & 3.39 \\ 1.80 & - & 2.59 \\ 1.00 & - & 1.79\end{array}$

\section{Interpretation}

Exceeds quality standard

Practices and procedure satisfy quality standard

Planned practices and procedures are fully implemented and aligned

Beginning structures and mechanism are in place

No evidence

Table 1 shows the overall extent on school management among schools across the six congressional districts. It is evident that both groups of respondents are in the opinion that practices and procedure in all six districts satisfy the quality standard.

Looking closely at the four factors, District 1 showed prominent scores in Curriculum Learning (4.13). This may imply that learning systems are regularly and collaboratively monitored by the community and school management in this district for growth and development of learners. Furthermore, this district develops a better organizational structure that also ensures the implementation of its policies for the development of its programs. In the same way, the district's results also corroborated with the involvement of the schools in the district which fared well with RAFI's Seal of Excellent Education (SEED) Program. The SEED is "an annual data-gathering, monitoring, evaluating and awards program which identified outstanding public elementary and secondary schools in Cebu using an evidence-based scorecard and was developed to engage, mobilize and capacitate the school governing councils in all public schools at the same time promoting best practices by featuring local initiatives that further education development".

District 4 rated highest in Leadership and Governance (4.14).The teachers and administrators perceived that the practices and procedure on leadership and governance satisfy the standard prescribed by the Department of Education. 
Concerning accountability and continuous improvement, Districts 4 (3.95) and 7(3.95) exhibited the highest score. According to the respondents, continuous enhancement of the community had been observed through management structures and mechanisms which are responsive to the needs and demands of the community.

It was further observed that District 7 performs highly (4.04) in the Management of Resources. This indicates that the school management personnel in District 7 know how to maximize the available means and resources at hand. This is validated by the respondents who mentioned that a series of dialogue for planning and resource programming were made accessible.

Overall, this means that the school administrators were able to fulfill their duties and responsibilities required by their position as school administrators, thus, making school management across all congressional districts at par with the Department of Education's (DepEd) quality standards.

Table 2. Overall Extent of School Milieu across Six Congressional Districts

\begin{tabular}{|c|c|c|c|c|c|c|c|c|c|c|c|c|}
\hline \multirow[b]{2}{*}{$\begin{array}{l}\text { School Milleu } \\
\text { Factors }\end{array}$} & \multirow[t]{2}{*}{$\begin{array}{l}\text { Congression } \\
\text { al Dsitricts }\end{array}$} & \multicolumn{2}{|c|}{$\underset{\substack{\text { s } \\
(n=36)}}{\text { Administrator }}$} & \multicolumn{2}{|c|}{$\begin{array}{c}\text { Teachers } \\
(n=438)\end{array}$} & \multicolumn{2}{|c|}{$\begin{array}{l}\text { Students } \\
(n=766)\end{array}$} & \multicolumn{2}{|c|}{$\begin{array}{c}\text { Parents } \\
(n=8,145)\end{array}$} & \multirow{2}{*}{$\begin{array}{l}\text { Reliabilit } \\
\mathbf{y}\end{array}$} & \multirow{2}{*}{$\begin{array}{c}\text { Overall } \\
\text { Mean }\end{array}$} & \multirow[t]{2}{*}{ Interpretation } \\
\hline & & $\begin{array}{c}\text { Mea } \\
\mathbf{n}\end{array}$ & $\underset{v}{\text { StDe }}$ & $\begin{array}{c}\text { Mea } \\
\mathbf{n}\end{array}$ & $\underset{\mathbf{v}}{\text { StDe }}$ & $\underset{\mathbf{n}}{\operatorname{Mea}}$ & $\underset{\mathbf{v}}{\text { StDe }}$ & $\begin{array}{c}\text { Mea } \\
\mathbf{n}\end{array}$ & $\underset{\mathbf{v}}{\text { StDe }}$ & & & \\
\hline \multirow[t]{6}{*}{ Context } & District 1 & 4.47 & 0.10 & 4.27 & 0.04 & 3.72 & 0.06 & 3.92 & 0.01 & 0.8253 & 4.09 & $\begin{array}{l}\text { Practices and procedure satisfy quality } \\
\text { standard }\end{array}$ \\
\hline & District 2 & 4.15 & 0.10 & 4.28 & 0.03 & & & 4.02 & 0.03 & 0.8518 & 4.15 & $\begin{array}{l}\text { Practices and procedure satisfy quality } \\
\text { standard }\end{array}$ \\
\hline & District 3 & 4.40 & 0.26 & 4.34 & 0.02 & & & 4.08 & 0.03 & 0.8775 & 4.27 & Exceeds quality standard \\
\hline & District 4 & 4.46 & 0.08 & 4.31 & 0.06 & 4.00 & 0.04 & 4.07 & 0.03 & 0.8017 & 4.21 & Exceeds quality standard \\
\hline & District 5 & 4.09 & 0.16 & 4.29 & 0.02 & 4.08 & 0.30 & 3.97 & 0.03 & 0.8788 & 4.11 & $\begin{array}{l}\text { Practices and procedure satisfy quality } \\
\text { standard }\end{array}$ \\
\hline & District 7 & 4.50 & 0.00 & 3.40 & 0.07 & & & 3.82 & 0.04 & 0.7368 & 3.91 & $\begin{array}{l}\text { Practices and procedure satisfy quality } \\
\text { standard }\end{array}$ \\
\hline \multirow[t]{6}{*}{ Massing } & District 1 & 4.46 & 0.11 & 4.23 & 0.03 & 3.61 & 0.09 & 3.97 & 0.03 & 0.8032 & 4.07 & $\begin{array}{l}\text { Practices and procedure satisfy quality } \\
\text { standard }\end{array}$ \\
\hline & District 2 & 3.84 & 0.07 & 4.23 & 0.06 & & & 4.04 & 0.03 & 0.8506 & 4.04 & $\begin{array}{l}\text { Practices and procedure satisfy quality } \\
\text { standard }\end{array}$ \\
\hline & District 3 & 4.42 & 0.33 & 4.45 & 0.04 & & & 4.07 & 0.02 & 0.8378 & 4.31 & Exceeds quality standard \\
\hline & District 4 & 4.48 & 0.24 & 4.29 & 0.03 & 4.01 & 0.07 & 4.10 & 0.01 & 0.7586 & 4.22 & Exceeds quality standard \\
\hline & District 5 & 4.07 & 0.08 & 4.25 & 0.04 & 3.98 & 0.22 & 3.94 & 0.03 & 0.8499 & 4.06 & $\begin{array}{l}\text { Practices and procedure satisfy quality } \\
\text { standard }\end{array}$ \\
\hline & District 7 & 4.50 & 0.00 & 3.36 & 0.06 & & & 3.75 & 0.02 & 0.7633 & 3.87 & $\begin{array}{l}\text { Practices and procedure satisfy quality } \\
\text { standard }\end{array}$ \\
\hline \multirow[t]{6}{*}{ Interface } & District 1 & 3.69 & 0.46 & 4.07 & 0.14 & 3.58 & 0.05 & 4.02 & 0.01 & 0.8350 & 3.84 & $\begin{array}{l}\text { Practices and procedure satisfy quality } \\
\text { standard }\end{array}$ \\
\hline & District 2 & 3.75 & 0.26 & 4.24 & 0.05 & & & 4.09 & 0.05 & 0.8827 & 4.03 & $\begin{array}{l}\text { Practices and procedure satisfy quality } \\
\text { standard }\end{array}$ \\
\hline & District 3 & 3.50 & 0.97 & 4.46 & 0.14 & & & 4.10 & 0.28 & 0.7803 & 4.02 & $\begin{array}{l}\text { Practices and procedure satisfy quality } \\
\text { standard }\end{array}$ \\
\hline & District 4 & 4.37 & 0.21 & 4.18 & 0.05 & 3.98 & 0.05 & 4.11 & 0.02 & 0.8153 & 4.16 & $\begin{array}{l}\text { Practices and procedure satisfy quality } \\
\text { standard }\end{array}$ \\
\hline & District 5 & 3.78 & 0.54 & 4.17 & 0.04 & 4.19 & 0.24 & 3.98 & 0.02 & 0.8813 & 4.03 & $\begin{array}{l}\text { Practices and procedure satisfy quality } \\
\text { standard }\end{array}$ \\
\hline & District 7 & 4.50 & 0.00 & 3.19 & 0.18 & & & 3.77 & 0.03 & 0.7922 & 3.82 & $\begin{array}{l}\text { Practices and procedure satisfy quality } \\
\text { standard }\end{array}$ \\
\hline \multirow[t]{6}{*}{ Way Finding } & District 1 & 3.93 & 0.08 & 3.91 & 0.03 & 3.60 & 0.11 & 3.88 & 0.06 & 0.8925 & 3.83 & $\begin{array}{l}\text { Practices and procedure satisfy quality } \\
\text { standard }\end{array}$ \\
\hline & District 2 & 3.77 & 0.16 & 4.04 & 0.09 & & & 4.03 & 0.05 & 0.9093 & 3.94 & $\begin{array}{l}\text { Practices and procedure satisfy quality } \\
\text { standard }\end{array}$ \\
\hline & District 3 & 3.62 & 0.69 & 4.30 & 0.07 & & & 4.05 & 0.07 & 0.8993 & 3.99 & $\begin{array}{l}\text { Practices and procedure satisfy quality } \\
\text { standard }\end{array}$ \\
\hline & District 4 & 4.26 & 0.12 & 4.06 & 0.05 & 3.92 & 0.07 & 4.05 & 0.11 & 0.8546 & 4.07 & $\begin{array}{l}\text { Practices and procedure satisfy quality } \\
\text { standard }\end{array}$ \\
\hline & District 5 & 3.51 & 0.13 & 4.09 & 0.05 & 4.11 & 0.25 & 3.94 & 0.05 & 0.9101 & 3.91 & $\begin{array}{l}\text { Practices and procedure satisfy quality } \\
\text { standard }\end{array}$ \\
\hline & District 7 & 4.50 & 0.00 & 3.19 & 0.04 & & & 3.80 & 0.06 & 0.8381 & 3.83 & $\begin{array}{l}\text { Practices and procedure satisfy quality } \\
\text { standard }\end{array}$ \\
\hline \multirow[t]{6}{*}{ Social Space } & District 1 & 4.23 & 0.13 & 4.24 & 0.05 & 3.55 & 0.12 & 4.01 & 0.08 & 0.8526 & 4.01 & $\begin{array}{l}\text { Practices and procedure satisfy quality } \\
\text { standard }\end{array}$ \\
\hline & District 2 & 3.83 & 0.14 & 4.13 & 0.03 & & & 4.13 & 0.49 & 0.7459 & 4.03 & $\begin{array}{l}\text { Practices and procedure satisfy quality } \\
\text { standard }\end{array}$ \\
\hline & District 3 & 4.27 & 0.07 & 4.49 & 0.04 & & & 4.07 & 0.03 & 0.9017 & 4.28 & Exceeds quality standard \\
\hline & District 4 & 4.54 & 0.01 & 4.24 & 0.02 & 4.02 & 0.09 & 4.15 & 0.22 & 0.7733 & 4.24 & Exceeds quality standard \\
\hline & District 5 & 3.74 & 0.26 & 4.07 & 0.16 & 4.13 & 0.21 & 4.02 & 0.34 & 0.8035 & 3.99 & $\begin{array}{l}\text { Practices and procedure satisfy quality } \\
\text { standard }\end{array}$ \\
\hline & District 7 & 4.50 & 0.00 & 3.30 & 0.03 & & & 3.64 & 0.78 & 0.6092 & 3.81 & $\begin{array}{l}\text { Practices and procedure satisfy quality } \\
\text { standard }\end{array}$ \\
\hline \multirow[t]{6}{*}{ Comfort } & District 1 & 3.89 & 0.33 & 3.86 & 0.19 & 3.56 & 0.10 & 3.73 & 0.12 & 0.6863 & 3.76 & $\begin{array}{l}\text { Practices and procedure satisfy quality } \\
\text { standard }\end{array}$ \\
\hline & District 2 & 3.79 & 0.10 & 3.81 & 0.31 & & & 3.84 & 0.18 & 0.6807 & 3.82 & $\begin{array}{l}\text { Practices and procedure satisfy quality } \\
\text { standard }\end{array}$ \\
\hline & District 3 & 4.00 & 0.54 & 4.24 & 0.27 & & & 3.88 & 0.11 & 0.7552 & 4.04 & $\begin{array}{l}\text { Practices and procedure satisfy quality } \\
\text { standard }\end{array}$ \\
\hline & District 4 & 4.13 & 0.11 & 4.00 & 0.18 & 3.96 & 0.08 & 3.92 & 0.14 & 0.6286 & 4.00 & $\begin{array}{l}\text { Practices and procedure satisfy quality } \\
\text { standard }\end{array}$ \\
\hline & District 5 & 3.67 & 0.22 & 3.84 & 0.11 & 3.89 & 0.15 & 3.74 & 0.13 & 0.6991 & 3.78 & $\begin{array}{l}\text { Practices and procedure satisfy quality } \\
\text { standard }\end{array}$ \\
\hline & District 7 & 4.33 & 0.41 & 3.18 & 0.06 & & & 3.51 & 0.07 & 0.5421 & 3.68 & $\begin{array}{l}\text { Practices and procedure satisfy quality } \\
\text { standard }\end{array}$ \\
\hline
\end{tabular}


Legend:

Mean Range

$\begin{array}{lll}4.20 & - & 5.00 \\ 3.40 & - & 4.19 \\ 2.60 & - & 3.39 \\ 1.80 & - & 2.59 \\ 1.00 & - & 1.79\end{array}$

\section{Interpretation}

Exceeds quality standard

Practices and procedure satisfy quality standard

Planned practices and procedures are fully implemented and aligned

Beginning structures and mechanism are in place

No evidence

Both Districts 3 and 4 have high ratings in all factors except Interface. Table 2 points out that with school milieu, only Districts 3 and 4 have exceeded the quality standards as specified by DepEd. These factors are context, massing and social space. The rest of the districts' scores which ranged between 3.68-4.16, disclosed that the school milieu on the RAFI built classrooms have gratified the quality standards mandated of an ideal school milieu.

Table 3. Overall Extent of School Environment Layout across Six Congressional Districts

\begin{tabular}{|c|c|c|c|c|c|c|c|c|c|c|c|c|}
\hline \multirow{2}{*}{$\begin{array}{l}\text { School } \\
\text { Environm } \\
\text { ent Layout } \\
\text { Factors }\end{array}$} & \multirow{2}{*}{$\begin{array}{l}\text { Congressional } \\
\text { Districts }\end{array}$} & \multicolumn{2}{|c|}{$\underset{\substack{\text { Administrato } \\
(n=36)}}{\text { As }}$} & \multicolumn{2}{|c|}{$\begin{array}{l}\text { Teachers } \\
(n=438)\end{array}$} & \multicolumn{2}{|c|}{$\begin{array}{l}\text { Students } \\
(n=766)\end{array}$} & \multicolumn{2}{|c|}{$\begin{array}{c}\text { Parents } \\
(n=8,145)\end{array}$} & \multirow{2}{*}{$\begin{array}{l}\text { Reliabil } \\
\text { ity }\end{array}$} & \multirow{2}{*}{$\begin{array}{c}\text { Overall } \\
\text { Mean }\end{array}$} & \multirow[t]{2}{*}{ Interpretation } \\
\hline & & $\begin{array}{c}\text { Mea } \\
\mathbf{n}\end{array}$ & $\begin{array}{c}\text { StD } \\
\text { ev }\end{array}$ & $\underset{\mathbf{n}}{\mathrm{Mea}}$ & $\begin{array}{c}\text { StD } \\
\text { ev }\end{array}$ & $\begin{array}{c}\text { Mea } \\
\text { n }\end{array}$ & $\begin{array}{c}\text { StD } \\
\text { ev }\end{array}$ & $\begin{array}{c}\text { Mea } \\
\mathbf{n}\end{array}$ & $\begin{array}{c}\text { StD } \\
\text { ev }\end{array}$ & & & \\
\hline Physical & District 1 & 4.19 & 0.33 & 4.03 & 0.08 & 3.65 & 0.14 & 3.93 & 0.10 & 0.8697 & 3.95 & Practices and procedure satisfy quality standard \\
\hline $\mathbf{F e}$ & District 2 & 3.84 & 0.20 & 4.10 & 0.14 & & & 4.06 & 0.29 & 0.8251 & 4.00 & Practices and procedure satisfy quality standard \\
\hline atu & District 3 & 3.50 & 0.29 & 4.38 & 0.10 & & & 4.07 & 0.21 & 0.8411 & 3.99 & Practices and procedure satisfy quality standard \\
\hline \multirow{3}{*}{ res } & District 4 & 4.39 & 0.26 & 4.16 & 0.13 & 4.05 & 0.14 & 4.07 & 0.06 & 0.8346 & 4.17 & Practices and procedure satisfy quality standard \\
\hline & District 5 & 4.00 & 0.51 & 3.98 & 0.14 & 4.08 & 0.21 & 3.93 & 0.03 & 0.8920 & 4.00 & Practices and procedure satisfy quality standard \\
\hline & District 7 & 4.50 & 0.00 & 3.17 & 0.10 & & & 3.61 & 0.06 & 0.8250 & 3.76 & Practices and procedure satisfy quality standard \\
\hline \multirow{6}{*}{$\begin{array}{l}\text { Outdoor } \\
\text { Areas }\end{array}$} & District 1 & 4.22 & 0.11 & 3.91 & 0.02 & 3.60 & 0.09 & 3.79 & 0.34 & 0.8479 & 3.88 & Practices and procedure satisfy quality standard \\
\hline & District 2 & 4.08 & 0.06 & 4.04 & 0.04 & & & 4.04 & 0.06 & 0.8725 & 4.06 & Practices and procedure satisfy quality standard \\
\hline & District 3 & 3.67 & 0.45 & 4.34 & 0.06 & & & 4.02 & 0.23 & 0.8196 & 4.01 & Practices and procedure satisfy quality standard \\
\hline & District 4 & 4.27 & 0.14 & 4.02 & 0.06 & 3.94 & 0.51 & 3.99 & 0.19 & 0.7664 & 4.05 & Practices and procedure satisfy quality standard \\
\hline & District 5 & 3.92 & 0.35 & 4.10 & 0.05 & 4.30 & 0.22 & 3.86 & 0.07 & 0.9007 & 4.05 & Practices and procedure satisfy quality standard \\
\hline & District 7 & 4.50 & 0.00 & 3.22 & 0.03 & & & 3.66 & 0.07 & 0.7999 & 3.79 & Practices and procedure satisfy quality standard \\
\hline Learning & District 1 & 3.87 & 0.36 & 4.00 & 0.08 & 3.69 & 0.11 & 3.89 & 0.25 & 0.9245 & 3.86 & Practices and procedure satisfy quality standard \\
\hline \multirow{5}{*}{$\begin{array}{c}\text { Environm } \\
\text { ents }\end{array}$} & District 2 & 3.75 & 0.14 & 3.97 & 0.08 & & & 4.04 & 0.11 & 0.9412 & 3.92 & Practices and procedure satisfy quality standard \\
\hline & District 3 & 3.55 & 0.50 & 4.27 & 0.13 & & & 4.05 & 0.11 & 0.9442 & 3.96 & Practices and procedure satisfy quality standard \\
\hline & District 4 & 4.23 & 0.13 & 3.99 & 0.11 & 3.97 & 0.22 & 4.05 & 0.14 & 0.9114 & 4.06 & Practices and procedure satisfy quality standard \\
\hline & District 5 & 3.64 & 0.24 & 3.97 & 1.03 & 4.08 & 0.27 & 3.91 & 0.05 & 0.9500 & 3.90 & Practices and procedure satisfy quality standard \\
\hline & District 7 & 4.50 & 0.00 & 2.98 & 0.09 & & & 3.57 & 0.06 & & 3.68 & Practices and procedure satisfy quality standard \\
\hline Social & District 1 & 3.64 & 0.47 & 3.75 & 0.07 & 3.64 & 0.14 & 3.62 & 0.11 & 0.9014 & 3.66 & Practices and procedure satisfy quality standard \\
\hline \multirow[t]{5}{*}{ Areas } & District 2 & 3.81 & 0.18 & 3.74 & 0.07 & & & 3.78 & 0.08 & 0.9032 & 3.78 & Practices and procedure satisfy quality standard \\
\hline & District 3 & 3.33 & 0.19 & 3.81 & 0.13 & & & 3.81 & 0.15 & 0.8892 & 3.65 & Practices and procedure satisfy quality standard \\
\hline & District 4 & 3.58 & 0.26 & 3.56 & 0.26 & 3.89 & 0.27 & 3.76 & 0.08 & 0.8218 & 3.70 & Practices and procedure satisfy quality standard \\
\hline & District 5 & 3.14 & 0.18 & 3.66 & 0.11 & 3.94 & 0.18 & 3.66 & 0.10 & 0.9126 & 3.60 & Practices and procedure satisfy quality standard \\
\hline & District 7 & 4.42 & 0.29 & 2.40 & 0.07 & & & 3.30 & 0.06 & 0.8327 & 3.37 & $\begin{array}{l}\text { Planned practices and procedures are fully } \\
\text { implemented and aligned }\end{array}$ \\
\hline Media & District 1 & 3.52 & 0.37 & 3.88 & 1.37 & 3.73 & 0.13 & 3.56 & 0.33 & 0.8553 & 3.68 & Practices and procedure satisfy quality standard \\
\hline Access & District 2 & 3.21 & 0.15 & 3.53 & 0.20 & & & 3.79 & 0.29 & 0.8335 & 3.51 & Practices and procedure satisfy quality standard \\
\hline \multirow[t]{4}{*}{ Social } & District 3 & 3.29 & 0.35 & 3.82 & 0.18 & & & 3.80 & 0.19 & 0.8755 & 3.64 & Practices and procedure satisfy quality standard \\
\hline & District 4 & 3.60 & 0.17 & 3.55 & 0.16 & 3.87 & 0.06 & 3.77 & 0.13 & 0.8456 & 3.70 & Practices and procedure satisfy quality standard \\
\hline & District 5 & 3.10 & 0.21 & 3.62 & 0.16 & 3.81 & 0.20 & 3.61 & 0.15 & 0.8903 & 3.53 & Practices and procedure satisfy quality standard \\
\hline & District 7 & 4.43 & 0.27 & 2.42 & 0.06 & & & 3.46 & 0.07 & 0.8667 & 3.44 & Practices and procedure satisfy quality standard \\
\hline Visual & District 1 & 4.20 & 0.12 & 4.05 & 0.06 & 3.60 & 0.09 & 3.92 & 0.02 & 0.8817 & 3.94 & Practices and procedure satisfy quality standard \\
\hline Appearanc & District 2 & 3.45 & 0.18 & 3.97 & 0.04 & & & 4.05 & 0.32 & 0.8834 & 3.82 & Practices and procedure satisfy quality standard \\
\hline \multirow[t]{4}{*}{ e } & District 3 & 3.53 & 0.35 & 4.26 & 0.09 & & & 4.07 & 0.31 & 0.8182 & 3.96 & Practices and procedure satisfy quality standard \\
\hline & District 4 & 4.26 & 0.16 & 4.10 & 0.07 & 3.97 & 0.02 & 4.09 & 0.32 & 0.7328 & 4.10 & Practices and procedure satisfy quality standard \\
\hline & District 5 & 3.40 & 0.07 & 4.02 & 0.07 & 3.78 & 0.20 & 3.94 & 0.31 & 0.8169 & 3.78 & Practices and procedure satisfy quality standard \\
\hline & District 7 & 4.50 & 0.00 & 2.89 & 0.09 & & & 3.62 & 0.61 & 0.8073 & 3.67 & Practices and procedure satisfy quality standard \\
\hline Degree of & District 1 & 3.97 & 0.27 & 4.23 & 0.86 & 3.79 & 0.17 & 3.89 & 0.08 & 0.8785 & 3.97 & Practices and procedure satisfy quality standard \\
\hline & District 2 & 3.77 & 0.09 & 3.92 & 0.21 & & & 4.08 & 0.06 & 0.8861 & 3.92 & Practices and procedure satisfy quality standard \\
\hline \multirow{4}{*}{ Security } & District 3 & 3.19 & 0.16 & 4.20 & 0.15 & & & 4.07 & 0.06 & 0.8875 & 3.82 & Practices and procedure satisfy quality standard \\
\hline & District 4 & 4.07 & 0.23 & 3.93 & 0.23 & 4.06 & 0.25 & 4.05 & 0.04 & 0.8229 & 4.02 & Practices and procedure satisfy quality standard \\
\hline & District 5 & 3.36 & 0.34 & 3.92 & 0.19 & 3.81 & 0.15 & 3.91 & 0.06 & 0.9131 & 3.75 & Practices and procedure satisfy quality standard \\
\hline & District 7 & 4.08 & 0.86 & 3.05 & 0.10 & & & 3.62 & 0.08 & 0.8363 & 3.59 & Practices and procedure satisfy quality standard \\
\hline
\end{tabular}

Legend:

Mean Range

$\begin{array}{lll}4.20 & - & 5.00 \\ 3.40 & - & 4.19 \\ 2.60 & - & 3.39 \\ 1.80 & - & 2.59 \\ 1.00 & - & 1.79\end{array}$

\section{Interpretation}

Exceeds quality standard

Practices and procedure satisfy quality standard

Planned practices and procedures are fully implemented and aligned

Beginning structures and mechanism are in place

No evidence

Table 3 illustrates that among the congressional district recipients, District 4 reveals the highest assessments on the factors affecting the school environment in the aspects of physical features, learning environments, media access, visual appearance, and degree of safety and security. It is important to note that it is in District 4 where most municipalities 
and schools were directly affected by the typhoon Haiyan in 2013. Hence, such rating is understandable. The result also shows that the respondents found the newly constructed buildings to be functional, safe and conducive to learning. District 2, on the other hand, is the highest both in outdoor and social areas.

In summary, Table 3 demonstrated that the RAFI school environment layout across the congressional districts regarding of physical features, outdoor areas, learning environments, social areas, media access, transition spaces and circulation routes, visual appearance, safety, and security had complied well enough with the set school environment norms of DepEd.

\section{Principal Component Analysis (PCA) on Factors Affecting the Schools Management among Schools in the Province of Cebu}

The results given below showed the Principal Component Analysis (PCA) on the impact of factors affecting the school management among schools in the province of Cebu. The Multivariate Principal Component Analysis (PCA) is a data mining or exploratory data analysis technique that can be used to determine the impact or weight contributions of each factor on the extent of school management among schools in the province of Cebu. Table 4 and 5 below shows the results.

Table 4. Eigenanalysis of the Correlation Matrix

\begin{tabular}{lllll}
\hline Eigenvalue & 3.4650 & 0.2996 & 0.1295 & 0.1059 \\
\hline Proportion & 0.866 & 0.075 & 0.032 & 0.026 \\
Cumulative & 0.866 & 0.941 & 0.974 & 1.000 \\
\hline
\end{tabular}

Results reveal that the first eigenvector or principal component represents $86.60 \%$ of the total variance. This finding is sufficient and reasonable enough to represent School Management in its four aspects of factors describing and affecting it, namely Leadership \& Governance Curriculum \& Learning Accountability \& Continuous Improvement and Management of Resources.

Table 5. Principal Component Analysis Results on Factors Affecting the School Management among Schools in the Province of Cebu

\begin{tabular}{lrrrr}
\hline Variables & PC1 & PC2 & PC3 & PC4 \\
\hline Leadership \& Governance & 0.493 & 0.624 & 0.365 & -0.484 \\
Curriculum \& Learning & 0.508 & 0.282 & -0.751 & 0.314 \\
Accountability \& Cont Imp & 0.488 & -0.695 & -0.144 & -0.508 \\
Management of Resources & 0.511 & -0.218 & 0.531 & 0.639 \\
\hline
\end{tabular}

Based on the results, as shown in Table 5, the extent of the factors that affect the School Management can be modeled by the equation given by:

Extent of School Management Index $=0.493$ Leadership and Governance +0.508 Curriculum and Learning +0.488 Accountability and Continuous Improvement + 0.511 Management of Resources

The model recommends that as any one of these factors increases, the extent of school management index also increases. This means that the extent of school management varies directly with the level of articulation of the factors above.

\section{Principal Component Analysis on Factors Affecting the Schools Milieu among Schools in the Province of Cebu}

Table 6 and 7 below reveal the Principal Component Analysis on the impact of factors affecting the school milieu among schools in the province of Cebu.

Table 6. Eigenanalysis of the Correlation Matrix

\begin{tabular}{lrrrrrr}
\hline Eigenvalue & 4.7132 & 0.6881 & 0.2250 & 0.1619 & 0.1192 & 0.0926 \\
\hline Proportion & 0.786 & 0.115 & 0.037 & 0.027 & 0.020 & 0.015 \\
Cumulative & 0.786 & 0.900 & 0.938 & 0.965 & 0.985 & 1.000
\end{tabular}

Results reveal that the first eigenvector or principal component represents $78.60 \%$ of the total variance. This finding is sufficient enough to represent the six aspects of factors that affect the School Milieu namely Context, Massing, Interface, Way Finding, Social Space, and Comfort. 
Table 7. Principal Component Analysis Results on Factors Affecting the School Milieu among Schools in the Province of Cebu

\begin{tabular}{lrrrrrr}
\hline Variables & PC1 & PC2 & PC3 & PC4 & PC5 & PC6 \\
\hline Context & 0.397 & 0.498 & 0.103 & -0.625 & -0.300 & -0.321 \\
Massing & 0.315 & -0.859 & 0.050 & -0.350 & -0.065 & -0.185 \\
Interface & 0.436 & 0.104 & 0.206 & -0.159 & 0.784 & 0.343 \\
Way Finding & 0.428 & 0.028 & 0.494 & 0.622 & -0.059 & -0.425 \\
Social Space & 0.441 & -0.014 & -0.017 & 0.149 & -0.521 & 0.715 \\
Comfort & 0.418 & 0.052 & -0.837 & 0.228 & 0.128 & -0.232
\end{tabular}

Based on the results as shown in Table 7, the index, regarding the extent of the factors that affect the School Milieu can be modeled by the equation given by the Extent of School Milieu Index $=0.397$ Context +0.315 Massing +0.436 Interface +0.428 Way Finding +0.441 Social Space +0.418 Comfort

The model suggests that if the social space (the ability of the school environment to accommodate diverse human needs) increases, the extent of school milieu index increases as well. Social space, on the other hand, has an impact in the school milieu while the massing (parts of the building that gives it form, meaning, and variety) revealed the least impact. Interface (meeting place where the inside of the building connects with the outside), wayfinding (ability for students, staff, teachers, and visitors to discern routes, traffic patterns or passageways in or around the building) and context (school building setting) have also a considerable impact in the school milieu. These results imply that the extent of school milieu varies directly with context, massing, interface, wayfinding, social space and comfort parameters. More specifically, school milieu is mainly characterized by social space and interface of the school building.

\section{Principal Component Analysis on Factors Affecting the School Environment among Schools in the Province of Cebu}

Tables 8 and 9 depict the Principal Component Analysis on the impact of factors affecting the school environment among schools in the province of Cebu.

Table 8. Eigenanalysis of the Correlation Matrix

\begin{tabular}{lrrrrrrr}
\hline Eigenvalue & 5.8498 & 0.4449 & 0.2331 & 0.1719 & 0.1564 & 0.0945 & 0.0495 \\
\hline Proportion & 0.836 & 0.064 & 0.033 & 0.025 & 0.022 & 0.013 & 0.007 \\
Cumulative & 0.836 & 0.899 & 0.933 & 0.957 & 0.979 & 0.993 & 1.000
\end{tabular}

Results in Table 8 show that, the first eigenvector or principal component represents $83.6 \%$ of the total variance which indeed is sufficient enough, to represent the School Environment on the areas of Physical Features, Outdoor Areas, Learning Environments, Social Areas, Media Access, Visual Appearance, and Degree of Safety \& Accuracy.

Table 9. Principal Component Analysis Results on Factors Affecting the School Environment among Schools in the Province of Cebu

\begin{tabular}{lrrrrrrr}
\hline Variables & PC1 & PC2 & PC3 & PC4 & PC5 & PC6 & PC7 \\
\hline Physical Features & 0.380 & -0.499 & 0.058 & 0.128 & -0.179 & -0.331 & -0.667 \\
Outdoor Areas & 0.371 & -0.466 & 0.418 & -0.349 & 0.328 & 0.436 & 0.225 \\
Learning Environments & 0.398 & -0.120 & -0.067 & -0.009 & -0.215 & -0.587 & 0.658 \\
Social Areas & 0.365 & 0.447 & 0.581 & 0.416 & -0.340 & 0.202 & -0.005 \\
Media Access & 0.375 & 0.467 & -0.012 & -0.085 & 0.708 & -0.310 & -0.190 \\
Visual Appearance & 0.378 & -0.108 & -0.600 & 0.535 & 0.134 & 0.411 & 0.107 \\
Degree of Safety \& Accuracy & 0.378 & 0.299 & -0.345 & -0.628 & -0.423 & 0.224 & -0.156 \\
\hline
\end{tabular}

Based on the results in Table 9, the index of the extent of the factors, that affects the school environment can be modeled by the equation given by the

Extent of School Environment Index $=0.380$ Physical Features + 0.371 Outdoor Areas + 0.398 Learning Environments + 0.365 Social Areas + 0.375 Media Access + 0.378 Visual Appearance + 0.378 Degree of Safety and Accuracy

The model implies that if any of these factors namely Physical Features, Outdoor Areas, Learning Environments, Social 
Areas, Media Access, Visual Appearance, and Degree of Safety \& Security increases, the extent of school environment index increases as well. That is, the extent of school environment varies directly with the extent of conditions of the factors mentioned above. However, among all factors, the learning environment revealed to have the highest impact on the school environment than the other six (6) factors.

\section{The Impact of School Milieu on the School Management among Schools in the Province of Cebu}

The following regression analysis results determine the effect of the extent of factors affecting school milieu on school management of administrators among schools in the Province of Cebu. Hence, the results of which establish the predictive ability of the school milieu factors as possible antecedents of the administrators' effectiveness in school management.

Table 10. Analysis of Variance Results

\begin{tabular}{lrrrrr}
\hline Source & DF & Adj SS & Adj MS & F-Value & P-Value \\
\hline Regression & 1 & 569.4 & 569.40 & 20.05 & 0.000 \\
$\quad$ Extent of School Milieu & 1 & 569.4 & 569.40 & 20.05 & 0.000 \\
Error & 80 & 2272.2 & 28.40 & & \\
Total & 81 & 2841.6 & & & \\
\hline
\end{tabular}

Model Summary

$\begin{array}{ccrr}\text { S } & \text { R-sq } & \text { R-sq(adj) } & \text { R-sq(pred) } \\ 5.32935 & 20.04 \% & 19.04 \% & 14.66 \%\end{array}$

Table 11. Coefficients

\begin{tabular}{lrrrrr}
\hline Term & Coef & SE Coef & T-Value & P-Value & VIF \\
\hline Constant & 25.63 & 6.19 & 4.14 & 0.000 & \\
Extent of School Milieu & 0.547 & 0.122 & 4.48 & 0.000 & 1.00 \\
\hline
\end{tabular}

Regression Equation Model:

Extent of School Management $=25.63+0.547$ Extent of School Milieu

The results show that the extent of school milieu significantly predicted the extent of school management among schools in the province of $\operatorname{Cebu}(\beta=0.547, t=4.48, p=0.000)$. The extent of school milieu also explained a very significant proportion of variance in the effectiveness of school management $\left(r^{2}=20.04 \%, F(1,80)=20.05, p=0.000\right)$. That is, for every unit increase in the extent of school milieu, a rise of 0.547 on the effectiveness of school management among schools occurs.

School milieu, therefore, influences the level of effectiveness of administrators' school management. Consequently, a robust school milieu among schools in the province of Cebu will yield superior school management capabilities of its administrators. This means that an improved school milieu has a direct effect on the administrators' enhanced school management skills.

The Impact of School Environment on the School Management among Schools in the Province of Cebu

Table 12. Analysis of Variance Results

\begin{tabular}{lrrrrr}
\hline Source & DF & Adj SS & Adj MS & F-Value & P-Value \\
\hline Regression & 1 & 495.0 & 495.02 & 16.88 & 0.000 \\
$\quad$ Extent of School Environment & 1 & 495.0 & 495.02 & 16.88 & 0.000 \\
Error & 80 & 2346.5 & 29.33 & & \\
Total & 81 & 2841.6 & & & \\
\hline
\end{tabular}

Model Summary

$\begin{array}{clll}\text { S } & \text { R-sq } & \text { R-sq(adj) } & \text { R-sq(pred) } \\ 5.41589 & 17.42 \% & 16.39 \% & 12.47 \%\end{array}$


Table 13. Coefficients

\begin{tabular}{lrrrrr}
\hline Term & Coef & SE Coef & T-Value & P-Value & VIF \\
\hline Constant & 29.13 & 5.90 & 4.94 & 0.000 & \\
Extent of School Environment & 0.3143 & 0.0765 & 4.11 & 0.000 & 1.00 \\
\hline
\end{tabular}

Regression Equation Model:

Extent of School Management $=29.13+0.3143$ Extent of School Environment

The findings revealed that the extent of school environment significantly predicted the extent of school management among schools in the province of $\operatorname{Cebu}(\beta=0.3143, t=4.11, p=0.000)$. The extent of the school environment also explained a significant proportion of variance in the effectiveness of school management $\left(r^{2}=17.42 \%, F(1,80)=16.88, p=0.000\right)$. That is, for every unit increase of the extent of the school environment, a rise by 0.3143 on school management effectiveness among schools administrators are expected. This result means that the school environment moderately influences the administrators' school management effectiveness.

Students' Academic Performance among Schools with Classrooms Constructed/Rehabilitated by RAFI in the Province of Cebu from 2004 to 2016

Table 14. The Academic Performance of Students among Schools with Classrooms Constructed/Rehabilitated by RAFI in the Province of Cebu from 2004 to 2016

\begin{tabular}{cccl}
\hline $\begin{array}{c}\text { Range of Academic Performance } \\
\text { in Terms of Grades }\end{array}$ & $\begin{array}{c}\text { Frequency of } \\
\text { Students }\end{array}$ & Percentage & Categorical Equivalent \\
\hline 92 and Above & 46 & 11.03 & Excellent \\
$84-91$ & 200 & 47.96 & Very Satisfactory \\
$76-83$ & 167 & 40.05 & Satisfactory \\
$68-75$ & 3 & 0.72 & Beginner \\
67 and Below & 1 & 0.24 & Needs Improvement \\
Total & 417 & 100.00 & \\
\hline
\end{tabular}

Table 14 shows the academic performance of students occupants in RAFI classrooms. It can be noted that their academic performance improved and that majority performed well enough as projected by the $47.96 \%$ categorical equivalent.

On the other hand, it can also be deduced that the academic performance of students in RAFI constructed classrooms ranged from satisfactory $(40.5 \%)$ to very satisfactory $(47.96 \%)$ as shown in the table. Worth noting as well, is that $11.03 \%$ of these students are academically excellent performers.

The aforementioned categorical results reveal that, indeed, in this particular categorical descriptive analysis and interpretation, the RAFI constructed classrooms contributed much to the academic performance of students utilizing it. These results, however, will still be subjected to an advanced statistical treatment, in an attempt to find its resolve, if indeed RAFI-SRP concerning school milieu and school environment has an impact on the students' academic performance.

The Impact of School Milieu on Academic Performance of Students among Schools with Classrooms Rehabilitated/Constructed in the Province of Cebu 
Table 15. Analysis of Variance Results

\begin{tabular}{lrrrrr}
\hline Source & DF & Adj SS & Adj MS & F-Value & P-Value \\
\hline Regression & 6 & 234.6 & 39.101 & 1.57 & 0.153 \\
Context & 1 & 107.7 & 107.698 & 4.33 & 0.038 \\
Massing & 1 & 5.8 & 5.783 & 0.23 & 0.630 \\
Interface & 1 & 6.1 & 6.085 & 0.24 & 0.621 \\
Way Finding & 1 & 0.0 & 0.009 & 0.00 & 0.985 \\
Social Space & 1 & 168.2 & 168.241 & 6.76 & 0.010 \\
Comfort & 1 & 14.8 & 14.804 & 0.59 & 0.441 \\
Error & 793 & 19746.5 & 24.901 & & \\
$\quad$ Lack-of-Fit & 579 & 14853.6 & 25.654 & 1.12 & 0.161 \\
$\quad$ Pure Error & 214 & 4892.9 & 22.864 & & \\
Total & 799 & 19981.1 & & & \\
\hline
\end{tabular}

Model Summary

$\begin{array}{ccrc}\text { S } & \text { R-sq } & \text { R-sq(adj) } & \text { R-sq(pred) } \\ 4.99009 & 1.17 \% & 0.43 \% & 0.00 \%\end{array}$

Table 16. Coefficients

\begin{tabular}{lrrrrr}
\hline Term & Coef & SE Coef & T-Value & P-Value & VIF \\
\hline Constant & 84.475 & 0.937 & 90.11 & 0.000 & \\
Context & 0.1714 & 0.0824 & 2.08 & 0.038 & 3.51 \\
Massing & -0.0467 & 0.0969 & -0.48 & 0.630 & 3.56 \\
Interface & 0.0313 & 0.0633 & 0.49 & 0.621 & 3.59 \\
Way Finding & 0.0011 & 0.0582 & 0.02 & 0.985 & 3.74 \\
Social Space & -0.1999 & 0.0769 & -2.60 & 0.010 & 3.93 \\
Comfort & 0.087 & 0.113 & 0.77 & 0.441 & 3.05 \\
\hline
\end{tabular}

Context and social space surprisingly significantly predicted the student's grades among schools in the province of Cebu, the relationship, however, is weak

$$
\left(\beta_{1}=0.1714, t=2.08, p=0.038, \beta_{2}=-0.1999, t=-2.60, p=0.010\right) .
$$

This means that context (school building setting) has a weak significant direct impact on the students grade while a weak inverse effect for social space (the ability of the school environment to accommodate diverse human needs) on the students' academic performance as evidenced by a small coefficient of determination $r^{2}$ value of $1.17 \%$. The remaining four factors, namely massing, interface, wayfinding, and comfort revealed no impact on students' academic performance. That is, regardless of how good and satisfying are the assessments of these factors, the student's performance remains unaffected.

The school building setting or context factor weakly poses a significant impact on student's academic performance as it increases. This shows that the physical environment can affect students' comfort and their ability to learn to some extent. Comfort more likely affects students learning. Corollarily, the physical atmosphere can also affect the morale of the learners. According to Oni (1992) and Hallak (1990), physical facilities compose a strategic factor in the operation and functioning of an organization as they determine the excellent performance of any social organization or system including education. Physical environment plays a central role in any activity and makes it more conducive, successful and achievable.

On the other hand, social space as it increases will result in lesser academic performance. Too much consideration given 
to cater diversities will lead to complexities and will result in unachievable academic satisfaction due to lack of concentration. This is an indicator not to overdo things, which also play a vital function in the learning process.

Regression Analysis Results on Academic Performance of Students versus the Factors Affecting the School Environment, School Building Observation and Overall Impression for the School Environment among Schools in the Province of Cebu

Table 17. Analysis of Variance Results

\begin{tabular}{lrrrrr}
\hline Source & DF & Adj SS & Adj MS & F-Value & P-Value \\
\hline Regression & 9 & 559.1 & 62.119 & 2.53 & 0.007 \\
School Building Observation & 1 & 55.2 & 55.220 & 2.25 & 0.134 \\
Physical Features & 1 & 160.8 & 160.770 & 6.54 & 0.011 \\
Outdoor Areas & 1 & 1.1 & 1.118 & 0.05 & 0.831 \\
Learning Environments & 1 & 2.5 & 2.466 & 0.10 & 0.752 \\
Social Areas & 1 & 31.9 & 31.888 & 1.30 & 0.255 \\
Media Access & 1 & 4.6 & 4.645 & 0.19 & 0.664 \\
Visual Appearance & 1 & 92.7 & 92.671 & 3.77 & 0.053 \\
Degree of Safety and Security & 1 & 272.2 & 272.171 & 11.07 & 0.001 \\
Overall Impression & 1 & 34.6 & 34.583 & 1.41 & 0.236 \\
Error & 790 & 19422.0 & 24.585 & & \\
Lack-of-Fit & 596 & 14638.5 & 24.561 & 1.00 & 0.521 \\
Pure Error & 194 & 4783.6 & 24.658 & & \\
Total & 799 & 19981.1 & & & \\
\hline
\end{tabular}

Model Summary

$\begin{array}{clcc}\mathrm{S} & \mathrm{R} \text {-sq } & \mathrm{R} \text {-sq (adj) } & \text { R-sq (pred) } \\ 4.95831 & 2.80 \% & 1.69 \% & 0.64 \%\end{array}$

Table 18. Coefficients

\begin{tabular}{lrrrrr}
\hline Term & Coef & SE Coef & T-Value & P-Value & VIF \\
\hline Constant & 84.524 & 0.934 & 90.47 & 0.000 & \\
School Building Observ & -0.162 & 0.108 & -1.50 & 0.134 & 3.20 \\
Physical Features & 0.1850 & 0.0723 & 2.56 & 0.011 & 5.54 \\
Outdoor Areas & 0.0115 & 0.0537 & 0.21 & 0.831 & 2.77 \\
Learning Environments & -0.0136 & 0.0430 & -0.32 & 0.752 & 8.58 \\
Social Areas & -0.0679 & 0.0596 & -1.14 & 0.255 & 3.97 \\
Media Access & 0.0260 & 0.0598 & 0.43 & 0.664 & 4.83 \\
Visual Appearance & 0.1297 & 0.0668 & 1.94 & 0.053 & 3.00 \\
Degree of Safety and Security & 0.2275 & 0.0684 & 3.33 & 0.001 & 4.07 \\
Overall Impression & 0.157 & 0.132 & 1.19 & 0.236 & 2.05 \\
\hline
\end{tabular}

The regression analysis results revealed a minimal impact of the three aspects of the school environment, physical features, visual appearance and degree of safety and security 


$$
\left(\beta_{1}=0.1850, t=2.56, p=0.011, \beta_{2}=0.1297, t=1.94, p=0.053, \beta_{3}=0.2275, t=3.33, p=0.001 \text {, respectively }\right)
$$

with the corresponding coefficient of determination of $2.80 \%$. These factors have to do with the overall physical environment of the school building. Physical facilities are one of the stimulating factors that play a fundamental role in improving academic achievement in the school system. These include; school buildings, accommodation, classrooms, libraries, furniture, laboratories, recreational equipment, apparatus, and other instructional materials. Furthermore, their availability, relevancy, and sufficiency affect academic achievement positively. Taylor and Vlastos (2009) establish the relationship between the environment and design within the classroom from a theoretical perspective. They found that the physical environment of the classroom acts as "Silent curriculum." It means that classroom environmental design can facilitate and improve the learning process like the overt curriculum.

Poor and inadequate facilities affect the overall performance of the institutions. Sufficient facilities promote academic achievement and ensure to strengthen the overall institutional performance. While unattractive and old school buildings; cracked classroom walls and floors; lack of toilets; lack of desks and benches; lack of transport facility; lack of proper security system; lack of drinking water; lack of power supply; lack of playgrounds; lack of teaching staff; lack of sufficient classrooms; overcrowded classrooms; lack of educational technology; lack of first aids facility etc negatively affect academic achievement of the institutions. Therefore, it is proper to say that academic achievement has a close link to the availability of educational facilities (Hussain et al. 2012).

On the other hand, the degree of safety and security records a weak impact. While there have been only a few studies that examined the effects of school safety on students' academic abilities in school, these few studies have shown that school safety has a significant impact on academic achievement. Milam et al. (2010) posit that increasing perceived safety increased achievement on standardized math and reading tests from 16 to $22 \%$ for Baltimore elementary schools. Schools that have lower academic scores tend to have a student population that does not feel safe within their schools. Conversely, schools that have higher academic scores tend to have a student population that feels safe within their schools.

Perceptions of school safety can be affected by a myriad of factors. The presence of gangs and drug problems can negatively affect students' perceptions of school safety (Schreck \& Miller, 2003). Security, surveillance, and other preventative measures can increase or decrease students' feelings of safety within their schools depending on how they are implemented (Kitsantas et al., 2004; Schreck \& Miller, 2003). Schools that have smaller student populations are more likely to foster feelings of safeness than schools with larger school populations (Bowen et al. 2000). Where the school is located also has profound implications for perceptions of school safety. Bosworth, K., Ford, L., \& Hernandez, D. (2011), for instance, found in their study that neighborhood surroundings have a very influential effect on students' perceptions of safety. Schools that are located in neighborhoods that have high poverty and high crime rates have been shown to have a negative influence on perceptions of school safety (Laub \& Lauritsen, 1998).

This study concurs with Bowen (1999) that finds an association between perceived school safety and academic achievement in middle school and high schools. However, this study does not take into account important variables like the degree of disciplinary problems in a school, the attendance of the respondents, and the degree of teacher professionalism and experience in a respondent's school. Thus, some of these variables may have been partially controlled for by taking into account the locations of where the respondents attended school (urban, rural, or suburban location).

\section{Conclusion}

The Ramon Aboitiz Foundation Incorporated School Rehabilitation Program (RAFI-SRP) affects the level of effectiveness of administrators in school management, which means that an enhancement in school milieu, improved the school management capabilities. Likewise, school environment influences the effectiveness of administrators in school management.

RAFI-SRP building specifics such as context and social space surprisingly pose a relatively weak but significant impact on student's academic performance. More specifically, the context (school building setting) has a direct, minimal significant impact, while a weak inverse relationship is attributed for social space, on students' academic performance.

RAFI-SRP has a modest influence on the academic performance of students among the community beneficiaries as a whole. This may be owed to the fact that academic performance is multifactorial, such that the interplay of factors may be intrinsic and extrinsic. RAFI-SRP is just one amongst the extrinsic factors to the learners' academic performance to contend with.

\section{Recommendation}

The researchers recommend a closer look at the instruction aspect of the teaching-learning process as a possible focus in future researches. On the other hand, it is recommended that a separate study is conducted on the classrooms/ schools 
constructed by RAFI 2014 onwards, vis a vis school's instruction quality. This is to ascertain if indeed such construction has a strong impact on the student's learning performance. In the same manner, the RAFI-SRP new buildings by that time could have been fully utilized given ample time to ripen its operation and utilization as the new building was constructed and turned over in 2014-2015.

\section{Acknowledgement}

This work was solely funded by the Ramon Aboitiz Foundation, Incorporated (EDU Grant CP2 2017). And, the copyright of this study belongs to Ramon Aboitiz Foundation Incorporated (RAFI).

\section{References}

Baker \& Bernstein. (2012). The Impact of School Buildings on student health and performance.

Bartlett, J. E., Kotrlik, J. W., \& Higgins, C. C. (2001). Organizational Research: Determining Appropriate Sample Size in Survey Research. Information Technology, Learning, and Performance Journal, 19, 43-50.

Bosworth, K., Ford, L., \& Hernandez, D. (2011). School Climate Factors Contributing to Student and Faculty Perceptions of Safety in Select Arizona Schools. Journal of School Health, 81(4), 194-201. https://doi.org/10.1111/j.1746-1561.2010.00579.x

Bowen, G. L., Bowen, N. K., \& Richman, J. M. (2000, April). School size and middle school students' perceptions of the school environment. Children \& Schools, 22(2), 69-82. https://doi.org/10.1093/cs/22.2.69

Bowen, N. K., \& Bowen, G. L. (1999). Effects of crime and violence in neighborhoods and schools on the school behavior and performance of adolescents. Journal of Adolescent Research, 14(3), 319-342. https://doi.org/10.1177/0743558499143003

Cash, C., \& Twiford, T. (2009). Improving student achievement and school facilities in a time of limited funding. International Journal of Educational Leadership Preparation, 4(2), 1-9.

Corcoran, T. B., Walker, L. J., \& White, J. L. (1988). Working in Urban Schools. Washington, DC: Institute for Educational Leadership. Retrieved from website: http://eric.ed.gov/ERICWebPortal/detail?accno=ED99356

Dalton, W. T. (1951). Classroom atmosphere reflects the quality of learning. Educational Leadership, 8(7), 429-433. Retrieved from http://www.ascd.org/ASCD/pdf/journals/ed_lead/el_195104_dalton.pdf

Department of Education. (2012). Department of Education (DepEd) Order No. 83, series 2012 - Implementing Guidelines on the Revised School-Based Management (SBM) Framework, Assessment Process and Tool (APAT). Retrieved from http://www.deped.gov.ph/2012/11/29/do-83-s-2012-implementing-guidelines-on-the-revised-school-based-manage ment-sbm-framework-assessment-process-and-tool-apat/

Hallak, J. (1990). Investing in the future: Setting educational priorities in the developing world. Paris: Pergonion Press.

Hopland, A. O., \& Nyhus, O. H. (2016). Learning environment and student effort. International Journal of Educational Management, 30(2), 271-286. https://doi.org/10.1108/IJEM-05-2014-0070

Hussain, I., Ahmad, M., Ahmad, S., Suleman, Q., Din, M. Q., \& Khalid, N. (2012). A study to investigate the availability of educational facilities at the secondary school level in District Karak. Language in India: Strength for Today and Bright Hope for Tomorrow, 12(10), 234-250.

Kitsantas, A., Ware, H. W., \& Martinez-Arias, R. (2004). Students' perceptions of school safety: Effects by community, school environment, and substance use variables. The Journal of Early Adolescence, 24(4), 412-430. https://doi.org/10.1177/0272431604268712

Laub, J. H., \& Lauritsen, J. L. (1998). The interdependence of school violence with neighborhood and family conditions. In D. S. Elliott, B. A. Hamburg, \& K. R. Williams (Eds.), Violence in American schools: A new perspective (pp. 127-155). New York, NY: Cambridge University Press.

Lave, J., \& Wenger, E. (1990). Situated learning: Legitimate peripheral participation. Cambridge, UK: Cambridge University Press.

Milam, A. J., Furr-Holden, C. D. M., \& Leaf, P. J. (2010). Perceived school and neighborhood safety, neighborhood violence and academic achievement in urban school children. The Urban Review, 42(5), 458-467. https://doi.org/10.1007/s11256-010-0165-7

Oni, J. O. (1992). Resource and resource utilization as correlates of school academic performance (Unpublished doctoral dissertation) University of Ibadan, Ibadan, Nigeria. 
Piggot, A. (2012). Standards of School Buildings... or... Ignorance Is Bliss? Part 1. Education in Science, 14-15.

Sanoff, H. (2001). School building assessment methods. Wahington, DC: National Clearinghouse for Educational Facilities. Retrieved from http://www.ncef.org/pubs/sanoffassess.pdf

Schreck, C. J., \& Miller, J. M. (2003). Sources of fear of crime at school: What are the relative contribution of disorder, individual characteristics, and school security? Journal of School Violence, 2(4), 57-79. https://doi.org/10.1300/J202v02n04_04

Taniguchi, K., \& Hirakawa, Y. (2016). Dynamics of community participation, student achievement, and school management: the case of primary schools in a rural area of Malawi.

Taylor, A. P., \& Enggass, K. (2009). Linking architecture and education: Sustainable design for learning environments. Albuquerque: University of New Mexico Press.

Yeoman, B. (2012). Special report: Rebuilding America's schools. Parade Magazine. Retrieved from http://www.parade.com/news/2012/08/12-rebuilding-americas-schools.html

\section{Copyrights}

Copyright for this article is retained by the author(s), with first publication rights granted to the journal.

This is an open-access article distributed under the terms and conditions of the Creative Commons Attribution license which permits unrestricted use, distribution, and reproduction in any medium, provided the original work is properly cited. 